\title{
TITLE: Colon Hypersensitivity to Distension, Rather Than Excessive Gas \\ Production, Produces Carbohydrate-related Symptoms in Individuals with Irritable Bowel Syndrome
}

AUTHORS: Giles Major ${ }^{1}$, Sue Pritchard², Kathryn Murray², Jan Paul Alappadan², Caroline Hoad $^{2}$, Luca Marciani ${ }^{1}$, Penny Gowland ${ }^{2}$, Robin Spiller ${ }^{1}$

1. Nottingham Digestive Diseases Centre, University of Nottingham UK

2. Sir Peter Mansfield Imaging Centre, University of Nottingham UK

GRANT SUPPORT: This is a summary of independent research funded by the National Institute for Health Research (NIHR)'s Nottingham Digestive Diseases Biomedical Research Unit. The views expressed are those of the authors and not necessarily those of the NHS, the NIHR or the Department of Health.

\section{DISCLOSURES:}

RS has received research funding from Lesaffre and Ironwood and free drug for clinical trial from Norgine and Falk Pharma. He has also acted on Advisory Boards for Almirall, Astellas, Yuhan Corporation and Danone. None of the authors have a conflict of interest to declare.

AUTHOR CONTRIBUTIONS: Study concept and design - GM, CH, LM, PG, RS; acquisition of data - GM, SP, JP, CH, LM, PG; analysis and interpretation of data - GM, SP, KM, CH, LM, PG, RS; drafting of the manuscript - GM, SP, RS, LM; critical revision of the manuscript for important intellectual content - All. 


\section{CORRESPONDENCE:}

Professor Robin Spiller

Address:

Nottingham Digestive Diseases Centre

E Floor West Block

Queens Medical Centre

Nottingham NG7 2UH

email: robin.spiller@nottingham.ac.uk

tel: $\quad+44(0) 1158231090$

fax: $\quad+44(0) 1158231409$

\begin{tabular}{|l|l|}
\hline au & Arbitrary units \\
\hline AUC & Area Under the Curve \\
\hline CSS & Composite Symptom Score \\
\hline$\Delta C S S$ & Change in Composite Symptom Score \\
\hline DP & Degree of polymerisation \\
\hline FODMAPs & Fermentable oligosaccharides, disaccharides, monosaccharides and polyols \\
\hline GI & Gastrointestinal \\
\hline$H_{2}$ & Hydrogen gas \\
\hline HADS & Hospital Anxiety and Depression Scale \\
\hline IBS & Irritable Bowel Syndrome \\
\hline MRI & Magnetic Resonance Imaging \\
\hline PHQ & Patient Health Questionnaire \\
\hline ppm & Parts per million \\
\hline Q25, Q75 & Lower and upper quartile \\
\hline SBWC & Small bowel water content \\
\hline SD & Standard Deviation \\
\hline
\end{tabular}




\section{ABSTRACT:}

Background \& Aims: Poorly digested, fermentable carbohydrates may induce symptoms of irritable bowel syndrome (IBS), via unclear mechanisms. We performed a randomized trial with magnetic resonance imaging (MRI) analysis to investigate correlations between symptoms and changes in small and large bowel contents following oral challenge.

Methods: We performed a 3-period crossover study of 29 adult patients with IBS (based on Rome III criteria, with symptoms of abdominal pain or discomfort for at least 2 days/week) and reported bloating. In parallel we performed the same study of 29 healthy individuals (controls). Studies were performed in the United Kingdom from January 2013 through February 2015. On 3 separate occasions (at least 7 days apart), subjects were given a 500 $\mathrm{ml}$ drink containing $40 \mathrm{~g}$ of carbohydrate (glucose in the first period, fructose in the second, and inulin in the third, in a random order). Levels of breath hydrogen were measured and intestinal content was assessed by MRI before and at various time points after consumption of each drink. Symptoms were determined based on subjects' responses to the Hospital Anxiety and Depression Scale questionnaire and the Patient Health Questionnaire-15. The primary endpoint was whether participants had a clinically important symptom response during the 300 minutes following consumption of the drink.

Results: More patients with IBS reached the pre-defined symptom threshold after intake of inulin $(13 / 29)$ or fructose $(11 / 29)$ than glucose $(6 / 29)$. Symptoms peaked sooner after intake of fructose than inulin. Fructose increased small bowel water content in both patients and controls whereas inulin increased colonic volume and gas in both. Fructose and inulin increased breath hydrogen levels in both groups, compared to glucose; fructose produced an earlier increase than inulin. Controls had lower symptom scores during the period after drink consumption than patients with IBS, despite similar MRI parameters and breath hydrogen responses. In patients who reached the symptom threshold after inulin intake, peak symptom intensity correlated with peak colonic gas $(r=0.57 ; P<0.05)$. Changes in MRI 
features and peak breath hydrogen levels were similar in patients who did and did not reach symptom threshold.

Conclusions: Patients with IBS and healthy individuals without IBS (controls) have similar physiological responses following intake of fructose or inulin; patients more frequently report symptoms after inulin than controls. In patients with a response to inulin, symptoms relate to levels of intra-luminal gas, but peak gas levels do not differ significantly between responders, non-responders or controls. This indicates that colonic hypersensitivity to distension, rather than excessive gas production, produces carbohydrate-related symptoms in patients with IBS. Clinicaltrials.gov no: NCT01776853

KEY WORDS: FODMAP; bloating; fermentation; MRI 


\section{Background \& Aims}

Most patients with the irritable bowel syndrome (IBS) report that certain foods can exacerbate symptoms ${ }^{1}$. Identifying the components of food responsible for symptoms has proved difficult. The concept of food allergy was popular but is now recognised to affect only a small proportion of IBS sufferers in most general gastroenterology clinics ${ }^{2}$. The role of bran and fibre has been debated for many years with evidence of benefit in constipation ${ }^{3}$ but aggravation of other symptoms such as flatulence ${ }^{4}$. More recently there has been renewed interest in a role for short-chain, poorly digested, fermentable carbohydrates termed fermentable oligosaccharides, disaccharides, monosaccharides and polyols (FODMAPs).

Evidence of a link between dietary FODMAPs and symptoms comes from a double-blind crossover study exposing IBS patients to escalating intake of either glucose, fructose, oligofructose or a mixture of the latter two for up to 2 weeks $^{5}$. Fructose or oligofructose were significantly more likely than glucose to induce symptom recurrence. A subsequent crossover study found that patients reported fewer symptoms following a low FODMAP diet compared to a typical Australian diet ${ }^{6}$. A UK study found that low FODMAP dietary advice was more efficacious than standard dietary advice for IBS though it should be noted that this was not placebo controlled ${ }^{7}$. More recently a randomised comparison found no difference between a low FODMAP and an empirical "IBS diet" based on healthy eating patterns, low fat content and avoidance of beans, cabbage and onions ${ }^{8}$.

While evidence regarding efficacy continues to accrue, less work has been published on the mechanisms by which dietary changes may affect symptoms. FODMAPs are believed to induce symptoms by two principal mechanisms. The 'small bowel' hypothesis contends that unabsorbed, osmotically active carbohydrates draw water into the small bowel, as has been previously demonstrated with lactose in the context of lactase deficiency ${ }^{9}$. This causes distension with symptoms of bloating and discomfort. The increased small bowel water content also accelerates oro-caecal transit, reducing small bowel absorption. The 'large bowel' hypothesis proposes that rapid colonic fermentation of unabsorbed carbohydrates 
generates gas, distending the colon and causing flatulence, bloating and discomfort. Reports that some IBS patients have different microbiota to non-sufferers ${ }^{10,11}$, and that the microbiota changes with diet, ${ }^{12,13}$ suggest that the metabolic output of the microbiota such as hydrogen $\left(\mathrm{H}_{2}\right)$ and other gases may be important mediators in the symptom response to both FODMAPs and the low FODMAP diet. Equally, evidence for visceral hypersensitivity in a subset of IBS patients suggests that the same magnitude of stimulus will produce different degrees of symptom response in patients depending on their sensory threshold ${ }^{14}$.

Measurement of luminal physiology has previously relied on invasive techniques such as manometry or barostat, or imaging techniques requiring ionising radiation such as scintigraphy or computed tomography. Wireless capsules have added new insights ${ }^{15}$ but cannot provide concurrent recording from the whole intestine. Recent advances in magnetic resonance imaging (MRI) allow assessment of the luminal environment without disturbing the system under investigation or using ionising radiation, a particularly advantageous feature in the substantial proportion of IBS patients who are young and female ${ }^{16}$. Application of MRI to measurement of small bowel water content has been validated ${ }^{17}$ and used to demonstrate the osmotic effects of mannitol ${ }^{18}$ and lactulose ${ }^{19}$ in IBS patients and healthy volunteers. Assessment of colonic content is feasible but requires prior definition of its volume using approaches commonly applied to imaging of solid viscera ${ }^{20,21}$. Luminal gas has been assessed in both the small bowel22 and colon ${ }^{23}$.

A recent study used a combination of these approaches to assess the differential effects of two poorly digested/ absorbed carbohydrates, inulin and fructose, on intestinal content in healthy subjects ${ }^{23}$. Inulin, an osmotically inactive polysaccharide with a high degree of polymerisation (DP), was used to investigate exclusively large bowel effects and induced an increase in colonic gas not seen with glucose. The monosaccharide fructose is variably absorbed and osmotically active, and in this study led to a profound increase in small bowel water content compared to glucose. The study excluded volunteers with a history of bowel disorders so was unable to comment on any relationship between physiological responses to 
carbohydrate ingestion and symptoms. Volunteers were also predominantly male and younger than the typical IBS population.

The aim of the current study was to investigate whether poorly digested/ absorbed carbohydrates would lead to symptoms in more IBS patients than a glucose control, and which changes in intestinal content would correlate best with IBS symptoms. We also sought to look for objective differences in luminal content between patients and a parallel sample of volunteers without IBS, frequency-matched to the IBS sample for age and gender.

\section{Methods}

\section{Design}

A three-period, three treatment crossover trial recruiting patients with IBS and bloating (Patient study), with a parallel cohort of healthy volunteers without functional gastrointestinal symptoms (Volunteer study) following the same trial regimen._The studies were approved by the United Kingdom National Research Ethics Service and prospectively registered on www.clinicaltrials.gov (NCT01776853). The trial ran from January 2013 to February 2015. A prospective amendment to the primary endpoint is described below and was the only material change to the published protocol. All authors had access to the study data and reviewed and approved the final manuscript.

\section{Participants}

All participants were adults aged 18-65. Patients were included if they met Rome III research criteria for IBS, reporting symptoms of abdominal pain/ discomfort for at least two days per week, and reported bloating as a frequent symptom. We chose such patients since it is these symptoms which respond best to the low FODMAP diet and hence it is this group of patients where there is most interest in the impact of fructose and fructans. Volunteers were included if they reported symptoms no more than once per month. An upper age limit was used to limit any effect of undiagnosed diverticular disease. Exclusion criteria included gastrointestinal (GI) surgery other than resection of the appendix or gall bladder, inability to 
stop drugs known to alter GI motility for the duration of the study or contraindication to MRI scanning. No interventions were administered within 4 weeks of antibiotic use. Patients were recruited through tertiary and secondary clinics in Nottingham, from a departmental database of patients prepared to take part in research and through open advertisement including the department's social media presence. All clinic patients had undergone appropriate screening following current guidelines and had normal haematological and biochemical values, negative coeliac serology and normal colonoscopy and biopsy if diarrhoea was a feature. Bile acid malabsorption, lactose malabsorption and pancreatic insufficiency were excluded where relevant. Those recruited through other routes had all been given a positive diagnosis by their general practitioner having had symptoms for more than 6 months. All such candidates completed a 7-day stool and symptom diary in addition to the Rome III questionnaire. Volunteers were recruited in a similar manner.

\section{Interventions and Procedures}

The treatments administered were $500 \mathrm{~mL}$ drinks consisting of 40 grams of carbohydrate dissolved in water, flavoured with $25 \mathrm{~mL}$ lemon juice, to be consumed in 15 minutes. The carbohydrates used were: a) 'glucose' - dextrose monohydrate (DP = 2) sold as Dextrose Powder (Thornton \& Ross, Huddersfield, UK); b) 'fructose' - 100\% fructose (DP =1) sold as Fruit Sugar Pure Fructose (Holland \& Barrett, Nuneaton, UK); c) 'inulin' - Orafti ${ }^{\circledR} \mathrm{HP}, \geq 99 \%$ chicory-derived inulin (DP $\geq 23$ ) supplied initially as a gift by Orafti (Belgium) then purchased from Beneo (Mannheim, Germany).

At enrolment all participants completed two questionnaires: the Hospital Anxiety and Depression Scale (HADS) and the Patient Health Questionnaire (PHQ)-15 from which we calculated the PHQ-12 by excluding the 3 Gl-related questions ${ }^{24}$. Participants then attended 3 treatment days separated by at least one week to minimise any carryover effect.

Participants were asked to moderate their diet for a full day before a treatment day, avoiding onion, garlic, pickles, sauces and other UK specific foods known to be high in fructans. 
Honey, jams, chocolates and other foods known to be high in fructose were also excluded. Bakery and dairy alternatives were also recommended to reduce intake of non-glucose carbohydrates. Participants fasted from 8pm in the evening_and remained nil by mouth from waking on the treatment day. Treatment days took place at the Sir Peter Mansfield Magnetic Resonance Centre. 3 modes of assessment were used: symptom questionnaire, $\mathrm{H}_{2}$ breath testing and MRI. Non-alcoholic mouthwash was administered prior to any breath testing. All 3 assessments were performed prior to the test drink and at $0,60,120,180,240$ and 300 minutes after its consumption. Symptoms and breath $\mathrm{H}_{2}$ were also assessed at 30 and 90 minutes.

\section{Randomisation and Blinding}

All participants took all treatments in the crossover design. The order of treatment was allocated according to a schedule randomly generated without blocking using Stata v13 by a member of the department not directly involved in the study delivery. The randomisation schedule was held by the departmental team of healthcare support workers responsible for preparing drinks who were independent of the study. All drinks were made with boiled water and then chilled to ensure standardisation. Both investigators and participants were blind to the intervention on each day. Analysis of MRI data was undertaken blind to challenge drink, symptoms and breath $\mathrm{H}_{2}$ readings. All entries into the study database were made prior to unblinding.

\section{Endpoints}

The primary endpoint was defined as whether participants reached a threshold of a "clinically important symptom response' (yes/ no) during the 300 minutes following the drink.-Clinical response was measured by a Composite Symptom Score including 4 symptoms: gas/ flatulence; bloating; pain/ discomfort; diarrhoea. Diarrhoea was included to reflect a consensus that fermentable carbohydrates cause increased water content of stool through osmotic pressure. At each time point each symptom was scored as 0 (none); 1 (mild/ negligible); 2 (moderate/ annoying); 3 (severe/ disabling). This score reflected previously 
used scoring systems ${ }^{25-27}$, including those applied to assess the effect of fructose and inulin on GI symptoms ${ }^{28,29}$, and addressed patients' conceptual framework for IBS ${ }^{30}$.

The aim of the threshold was to avoid false assignment of importance to diurnal variation in the symptoms commonly experienced by both patients and the general population. We judged that a CSS $\geq 3$ would have face validity as clinical important. Review of data from our previous study ${ }^{23}$ in healthy volunteers found that $1 / 16$ met this threshold for each intervention used, making it a plausible cut-off to discriminate a signal due to the test drink from background noise._A study published during the trial reporting high levels of baseline symptoms in IBS patients ${ }^{31}$ demonstrated the need to measure the increase in symptoms, not just an absolute score. The threshold for the primary endpoint was therefore amended from an absolute score to a rise in CSS $(\Delta \mathrm{CSS}) \geq 3$.

Secondary endpoints addressed physiological effects of the drinks. Breath $\mathrm{H}_{2}$ was measured in parts per million (ppm) using the Gastrolyzer device (Bedfont, UK). MRI measurements included small bowel water content (SBWC, $\mathrm{mL}$ ), colonic volume $(\mathrm{mL})$ and colonic gas (arbitrary units, au). 'Symptom intensity' was measured using a composite of visual analogue scales $(0-100)$ for each symptom as described by Skoog et al. ${ }^{28}$. This second symptom measurement was used to provide more finely graded assessment for quantitative analysis.

\section{MRI data analysis}

All images were acquired using a whole-body 1.5T scanner (Achieva, Philips Medical System, Best, The Netherlands). The methods for measurement of SBWC and colonic volume have been described in detail previously ${ }^{17,20}$. The sigmoid colon, is normally collapsed and not included in the measurement of the descending colon, however in this study it was noted to often be markedly distended and so was measured by our usual technique, as has recently been shown to be feasible by other groups ${ }^{32}$. Ultimately $36 / 58$ individuals had a measurable sigmoid colon for at least one time point, and in 20/58 this was more than $50 \mathrm{~mL}$, values which were included in the total colon volume. Colonic gas was 
measured using the following objective algorithm: using the data acquired for colonic volume measurements, the MRI analyst selected an index region of interest within a clearly defined pocket of gas in the colon. This pocket provided a mean(M) and standard deviation (SD) to define objectively the image intensity of gas. Areas within the colon were defined as gas where 5 or more adjoining voxels had an intensity $\leq M+2 S D$. To reduce inter-observer variability a single experienced operator analysed all data from the small bowel $(\mathrm{KM})$ and colon (SP).

\section{Sample size and Statistical Analysis}

The primary efficacy comparison was the difference in proportion of patients meeting the primary endpoint ( $\triangle \mathrm{CSS} \geq 3$ ). Each test intervention (fructose, inulin) was compared to glucose control (McNemar's test).

Secondary endpoints were compared using the area under the curve (AUC) of the change from baseline in the period 0-300 minutes following the drink. Each test intervention was compared to glucose control. Paired differences were assessed for normality by the ShapiroWilk test, confirmation of which permitted analysis by paired t-test for each intervention against control. Exploratory comparisons between patients and controls were made by Chisquared test for the primary endpoint and unpaired t-test for other endpoints. All analyses were undertaken using SPSS version 20 (IBM, USA). Results are presented without correction for multiple comparisons.

The sample size was estimated in order to address the primary efficacy comparison in patients based on past literature and pilot data described earlier. It was predicted that $10 \%$ of patients would meet primary endpoint after glucose and that a response rate of $40 \%$ after fructose or inulin would be noteworthy. No data was available on within-person correlation. With power set as $80 \%$ and a significance level of 0.05 a range of within-person correlations were modelled suggesting a sample size of $23-35$. Allowing for withdrawal, a sample size 
of 35 in each study was estimated to allow collection of 30 complete MRI data sets, the criteria for participants to be included for analysis in this mechanistic study.

Given the variation between individual symptom profiles against time, further exploratory analysis to assess correlations between physiological variables and symptoms used the peak rise from baseline in symptom intensity.

\section{Results}

29 participants completed each study. Details of exclusions and withdrawals are included in the supplementary information. After assessment by the Rome III questionnaire 5 patients met criteria for diarrhoea-predominant IBS, 6 for constipation-predominant IBS and 18 for mixed type. The demographics and psychological scores of participants and volunteers were similar, other than somatisation scores, and are summarised in Table 1.

\section{Table 1: Demographics of participants in Patient and Volunteer studies}

\section{Primary endpoint}

$6 / 29(21 \%)$ patients reached the symptom threshold ( $\triangle \mathrm{CSS} \geq 3$ ) after the glucose control, a higher rate than predicted. $13 / 29(45 \%)$ reached symptom threshold after inulin, a significantly higher proportion than after glucose $(P<0.05)$. The response rate was also somewhat higher after fructose $(11 / 29,38 \%)$ but not significantly so. In patients who reached threshold median time to peak symptom intensity was shorter $(P=0.06)$ with fructose $(90$ mins, $n=11$ ) than with inulin (240 mins, $n=13$ ). Volunteer response rates were lower for inulin at 3/29 $(P<0.01)$ and $3 / 29$ for fructose $(P<0.05) .2 / 29$ reached symptom threshold for glucose which was not significantly less than in the patient cohort. The paired results for patients and controls are summarised in Table 2.

Table 2: Contingency tables of subjects reaching primary endpoint ( $\Delta$ CSS $\geq 3$ )

\section{Secondary endpoints}


One patient's colonic images were not interpretable because of extremely large amounts of gas throughout the study so these were excluded from analysis. Two patients did not record a breath hydrogen reading $>20$ ppm at any point during the trial, suggesting a lack of hydrogen-producing bacteria._Data for area under the curve of the change from baseline for each variable are presented by intervention in Table 3 with time course data in Figure 1. In both studies the AUC for breath $\mathrm{H}_{2}$ and colonic volume was higher for both fructose and inulin than for glucose. In both studies the AUC for SBWC was higher after fructose than glucose, while the AUC for colonic gas was higher after inulin. In patients colonic gas was also increased after fructose but to a lesser degree $(P<0.05)$.

AUC for symptom intensity was higher in patients after both interventions and in volunteers after inulin compared to glucose $(P<0.05)$. The degree of change in intensity of symptoms tended to be higher in patients than in_volunteers (Figure 1A).

The patterns of change in the physiological variables were similar for both patients and volunteers $(\mathrm{P}>0.1$ for all, Figure 1B-E). After inulin changes in all variables followed a similar time course. Time to peak breath $\mathrm{H}_{2}$ was significantly shorter $(\mathrm{P}<0.001)$ after fructose (mean 108 mins, 95\% Cl $84-132, n=58$ ) than after inulin (mean 266 mins, 95\% Cl $249-283, n=58$ ), although the magnitude of change was higher after inulin (Table 3). The time course of fructose-induced changes in SBWC followed the same pattern as breath $\mathrm{H}_{2}$ but peaked significantly $(\mathrm{P}<0.001)$ sooner (mean 44 mins, $95 \% \mathrm{Cl} 33-56, \mathrm{n}=58$ ). The decline in breath $\mathrm{H}_{2}$ after 120 minutes diverged from the increase in colonic volume and gas seen at the same time. Subjective assessment of MRI sequences measuring SBWC noted high water content in the colon (Figure 2) but this variable was not specifically measured as the signal from fluid entering the colon is transient and fades variably over time.

Table 3: Postprandial change from baseline for secondary endpoints (Mean \pm SEM) 
Figure 1: Time series of symptom intensity and physiological variables for each intervention in Patients and Volunteers.

Top - patient data; Bottom - volunteer data. Panel A: Symptom intensity; Panel B: Breath hydrogen; Panel C: Small bowel water content; Panel D: Colonic gas; E: Colonic volume.

Figure 2: Example of MRI analysis showing colonic water shortly after fructose A: unprocessed T2-weighted image; B: detectable free water after threshold applied; C: anatomical image for comparison

\section{Correlations between physiology and symptoms}

The data from patients who met primary endpoint were explored for correlations between peak rise from baseline in physiological parameters and peak rise in symptom intensity. For the 12 patients with symptoms after inulin and usable colonic data, the best correlate was peak rise in colonic gas (Pearson's $r=0.57, n=12, P<0.05$, Figure 3 ). No significant correlation was seen with SBWC $(r=-0.1, n=13)$ nor breath $\mathrm{H}_{2} \quad(r=0.26, n=13)$. In the 11 patients with symptoms after fructose correlations were weaker although both peak rise in SBWC and breath $\mathrm{H}_{2}$ showed a non-significant relationship $(r=0.51, n=11, P=0.11$ for both). Too few healthy volunteers met primary endpoint for meaningful analysis.

Figure 3: Correlation between peak rise in colonic gas and peak rise in symptom intensity after inulin $(n=12)$

Lastly, the inulin data were explored for differences in the peak rise in colonic gas between subjects who did and did not reach the symptom threshold after inulin. No difference was seen between patients who did and did not report symptoms. In contrast the few healthy volunteers who reached threshold after inulin had peak rises in colonic gas at the top of the range for all subjects. Changes in colonic volume followed a similar pattern in both studies. Individual data points are shown in Figure 4. For fructose, the only variable that showed 
some difference between subjects who did and did not report symptoms was their initial score on the PHQ-12 questionnaire, measuring somatisation ( $P<0.05$, Mann-Whitney). Responders to fructose had higher PHQ-12 scores (median 8, IQR 7 - 12) than did nonresponders (median 5, IQR $3-8$ ), where a normal score is defined as less than 7.

Figure 4: Comparison of peak rise in colonic content between those who did and did not meet symptom threshold ( $\Delta$ CSS $\geq 3$ )

A: Colonic gas; B: Colonic volume

\section{Discussion}

The study is the first to use MRI to compare the effects of poorly digested, fermentable carbohydrates on symptoms and physiology in IBS. The most notable findings were those relating to inulin. $40 \mathrm{~g}$ of inulin induced more patients to reach our pre-determined symptom threshold than did $40 \mathrm{~g}$ of glucose. Inulin induced greater increases in breath $\mathrm{H}_{2}$, colonic gas and colonic volume. The time courses of change in those physiological variables and symptom intensity were similar. In those who reported symptoms above threshold, peak rise in symptom intensity correlated with peak rise in colonic gas but not colonic volume or breath $\mathrm{H}_{2}$. We did not measure breath methane, another gas that may have contributed to colonic expansion, but in our experience only a small proportion of subjects produce only methane so this would be unlikely to alter our conclusions. However in IBS patients the magnitude of peak increases of gas on MRI in those who exceeded the symptom threshold had a similar range to those who did not. The study therefore offered no evidence that patients who reported symptoms produced more colonic gas than those who did not. Those patients with a higher peak in breath hydrogen were no more likely to report symptoms with inulin or fructose, in agreement with other studies suggesting that the utility of a breath test to predict response to dietary fructose exclusion is limited ${ }^{33,34}$. An alternative hypothesis would be that, 
as has been shown with lactose malabsorption ${ }^{35}$, the patients with symptoms had a lower sensory threshold for reporting.

Some healthy volunteers reported mild symptoms, principally flatulence, but few exceeded our pre-determined symptom threshold despite physiological changes similar to those seen in IBS. Those few that did had a peak rise in colonic gas that was at the top end of the range in the sample. This would be consistent with the concept that the colon normally accommodates gas without symptoms unless particularly large volumes are produced. Any trial to test this formally, with a similar event rate, would need to recruit a far larger sample.

The findings in relation to fructose are more complex to interpret. Firstly, the difference in symptom response to fructose and glucose was not as clear cut as for inulin. The event rate in the glucose control period was higher than predicted, and higher than the event rate in either the healthy volunteer pilot study ${ }^{23}$ or the parallel volunteer study reported here. Previous studies suggest that the colonic motor response to feeding is calorie dependent and would normally require a much bigger load of glucose than the $40 \mathrm{~g}$ we gave ${ }^{36}$. Exactly what stimulus the patients are responding to is therefore unclear. It may reflect expectation and an increased tendency in IBS patients to report symptoms without any differences in sensitivity, as described by Dorn et al. ${ }^{14}$. Previous studies have found higher symptom rates with fructose in IBS patients but any inference drawn about the mechanisms of effect of fructose requires the caveat that differences in the primary endpoint from glucose control may have been due to chance alone. The higher degree of somatisation, as reflected by the PHQ-12 scores, in patients who did and did not report symptoms supports the idea that somatisation is related to visceral hypersensitivity as Dorn et al reported. We did not formally assess visceral hypersensitivity using a barostat but such studies could further validate MRI as a non-invasive measure of hypersensitivity to the stimulus of food related intestinal distension, which is most likely the everyday stimulus which causes pain in IBS patients. 
The size and time course of changes in small bowel water (SBWC) were similar to the initial study of Murray et al. and were seen in both study groups. Also noted were significant increases in breath $\mathrm{H}_{2}$ and colonic volume. Breath $\mathrm{H}_{2}$ levels peaked after SBWC and remained mildly elevated for several hours, presumably reflecting the small amount of fructose that escapes absorption and enters the colon. Nevertheless the increase in colonic volume cannot be solely attributed to colonic gas and may include increased influx of ileal fluid associated with increased SBWC. However, none of the variables measured in the study correlated strongly with symptom intensity nor showed large differences between those who did and did not exceed the symptom threshold. Where present, symptoms after fructose did peak earlier than after inulin, suggesting that small bowel distension might cause symptoms albeit not a sufficiently large effect to detect a signal with the sample size used here.

As such this study provides no evidence that poorly digested carbohydrates generate symptoms directly through small bowel distension. If there is a subset of patients who suffer symptoms as a result of small bowel liquid distension, then this would likely be due to abnormal motor patterns leading to a failure to disperse the fluid load through the length of the small bowel, with consequent local distension. There is an inherent challenge to the generation of such evidence while excluding any effect from subsequent colonic mechanisms. The absorption of fructose varies considerably between patients which reduces the power of our study to show a link with symptoms. Further work using nonabsorbable sugars such as lactulose ${ }^{19}$ may be needed to tease out the role of the small bowel. Zhu et al. have recently reported on the response to lactose in a Chinese population with a high prevalence of lactose maldigestion, where rectal hypersensitivity assessed by barostat was associated with a higher odds ratio for bloating $(6.61,95 \% \mathrm{Cl} 1.75-25)$ than was hydrogen production $(2.19,95 \% \mathrm{Cl} 1.1-4.4)^{37}$. Our results are consonant with these findings. 
Our study had limitations. The diagnosis of IBS was confirmed in patients using a clinical standard of care, in keeping with the principle of making a positive diagnosis. Other GI disorders may present as IBS although UK data suggest that presentation with coeliac disease, colorectal cancer or inflammatory bowel disease in the year following diagnosis is a rare event ${ }^{38}$. There is increasing interest in the role of small intestinal bacterial overgrowth in IBS. All participants in the study underwent a de facto glucose hydrogen breath test during the study. None met local diagnostic criteria for overgrowth, set at a rise from baseline of $>20 \mathrm{ppm}$. One asymptomatic volunteer recorded a rise in breath hydrogen of 15ppm. Small bowel aspirate and culture is rarely performed in the UK unless there are signs of malabsorption such as anaemia which none of our patients had. Participants had lower scores for anxiety and depression than we have previously reported in patients largely recruited from secondary care ${ }^{24}$. This may reflect our recruitment procedures which included patients in primary care and those who responded to advertisement. Specialist referral may reflect failure of coping strategies for patients and non-specialists, which may be associated with worse mental health. Our sample may therefore be more reflective of IBS sufferers in the general population.

We did not account for variation in regular diet of participants, nor did we exclude patients who had used a low FODMAP diet. To do so may have biased our sample in either direction: such patients may be more likely to incur symptoms when exposed to FODMAPs, or less likely if dietary modification leads to alterations in their microbiota with consequent changes in its carbohydrate metabolism. The longitudinal interactions between diet, microbiota, physiology and symptoms are still being elucidated and were beyond the scope of this work.

The patient study was not powered to account for such a frequent symptom response to drinking a glucose solution. This is most likely a nocebo response since the patient information sheet made it clear that one or more of the solutions might induce symptoms. An advantage of the crossover design is that any effect is only attributed to the test drink in patients who did not report symptoms after glucose. The unusual circumstances of the MRI 
centre may have exacerbated psychological distress but no effect of visit order was identified. Nevertheless, future studies will need to account better for the glucose response rate when calculating their sample size. As this was the first MRI study on this topic in patients we used frequent scanning over an extended period and so resource constraints meant that it was not feasible to study large numbers of patients. Now we have provided information on the time scale of changes, future studies can use more efficient protocols to provide increased power to detect smaller differences in effect size.

In addition the reductionist approach taken, using carbohydrate solutions, may not induce the full range of physiological response seen with a food product. Food composition may alter interaction with the microbiota on arrival in the colon. Chronic exposure may lead to alterations in the microbiota and subsequent physiological response. MRI data are not available to assess changes occurring after 5 hours. Future studies will need to look at the effect of smaller, chronic changes in fructose and fructan intake in order to understand the role of diet over time.

The analysis reported here combines the small and large bowel into single units. Symptoms may also relate to changes at focal points in the bowel, such as the flexures and sigmoid colon, which was not captured by the regional assessments made. There may also be variation between IBS subtypes. While all patients reported bloating, they had different stool habits which may reflect differences in pathophysiology. While there may be common pathways underlying bloating and discomfort across the IBS spectrum, future studies should consider reducing heterogeneity by focusing on one subtype.

The strength of the study is the unique insight that it provides on the undisturbed luminal environment during periods of symptoms. Traditional methods for assessing visceral sensitivity rely on invasive techniques such as the barostat. Such techniques inevitably alter the psychological state of the subject so may indirectly, and indeed directly change the visceral response. No invasive device was used in this study: the only interventions were 
drinks, breath tests and scans._The double-blind crossover design reduced bias in the interpretation of the differential effects of the drinks. While no formal comparisons between the patient and volunteer studies are presented, the physiological response appeared to be similar between the groups and confirmed that the changes seen in our original paper in younger healthy volunteers also occurred in samples of volunteers of IBS patients with similar age and gender mix to the typical IBS population. Inulin reliably induced colonic distension and in the future may provide a standard stimulus with which to study visceral sensitivity.

In conclusion this study demonstrates a mechanism by which dietary carbohydrates that are poorly digested and then fermented induce symptoms of the irritable bowel syndrome. Patients experiencing symptoms did not have greater gas production or visceral distension but the degree of gas produced did correlate with the intensity of symptoms. This supports the hypothesis that visceral hypersensitivity is the reason that some IBS patients report symptoms after eating FODMAP-containing foods, and symptom relief when following a low FODMAP diet.

\section{References}

1. Bohn L, Storsrud S, Tornblom H, et al. Self-reported food-related gastrointestinal symptoms in IBS are common and associated with more severe symptoms and reduced quality of life. Am J Gastroenterol 2013;108:634-41.

2. Bischoff SC, Herrmann A, Manns MP. Prevalence of adverse reactions to food in patients with gastrointestinal disease. Allergy 1996;51:811-8.

3. Arffmann S, Andersen JR, Hegnhoj J, et al. The effect of coarse wheat bran in the irritable bowel syndrome. A double-blind cross-over study. Scand J Gastroenterol 1985;20:295-8.

4. Snook J, Shepherd HA. Bran supplementation in the treatment of irritable bowel syndrome. Aliment Pharmacol Ther 1994;8:511-4.

5. Shepherd S, Parker F, Muir J, et al. Dietary Triggers of Abdominal Symptoms in Patients With Irritable Bowel Syndrome: Randomized Placebo-Controlled Evidence. Clinical Gastroenterology and Hepatology 2008;6:765-771.

6. Halmos EP, Power VA, Shepherd SJ, et al. A diet low in FODMAPs reduces symptoms of irritable bowel syndrome. Gastroenterology 2014;146:67-75 e5. 
7. Staudacher HM, Whelan K, Irving PM, et al. Comparison of symptom response following advice for a diet low in fermentable carbohydrates (FODMAPs) versus standard dietary advice in patients with irritable bowel syndrome. Journal of Human Nutrition and Dietetics 2011;24:487-495.

8. Bohn L, Storsrud S, Liljebo T, et al. Diet low in FODMAPs Reduces Symptoms of Irritable Bowel Syndrome as Well as Traditional Dietary Advice: A Randomized Controlled Trial. Gastroenterology 2015.

9. Christopher NL, Bayless TM. Role of the small bowel and colon in lactose-induced diarrhea. Gastroenterology 1971;60:845-52.

10. Rajilić-Stojanović M, Biagi E, Heilig HGHJ, et al. Global and Deep Molecular Analysis of Microbiota Signatures in Fecal Samples From Patients With Irritable Bowel Syndrome. Gastroenterology 2011;141:1792-1801.

11. Jeffery IB, O'Toole PW, Ohman L, et al. An irritable bowel syndrome subtype defined by species-specific alterations in faecal microbiota. Gut 2012;61:997-1006.

12. Wu GD, Chen J, Hoffmann C, et al. Linking long-term dietary patterns with gut microbial enterotypes. Science 2011;334:105-8.

13. David LA, Maurice CF, Carmody RN, et al. Diet rapidly and reproducibly alters the human gut microbiome. Nature 2014;505:559-63.

14. Dorn SD, Palsson OS, Thiwan SI, et al. Increased colonic pain sensitivity in irritable bowel syndrome is the result of an increased tendency to report pain rather than increased neurosensory sensitivity. Gut 2007;56:1202-9.

15. Rao SS, Camilleri M, Hasler WL, et al. Evaluation of gastrointestinal transit in clinical practice: position paper of the American and European Neurogastroenterology and Motility Societies. Neurogastroenterol Motil 2011;23:8-23.

16. Marciani L. Assessment of gastrointestinal motor functions by MRI: a comprehensive review. Neurogastroenterology \& Motility 2011;23:399-407.

17. Hoad CL, Marciani L, Foley S, et al. Non-invasive quantification of small bowel water content by MRI: a validation study. Physics in Medicine and Biology 2007;52:69096922.

18. Marciani L, Cox EF, Hoad CL, et al. Postprandial Changes in Small Bowel Water Content in Healthy Subjects and Patients With Irritable Bowel Syndrome. Gastroenterology 2010;138:469-477.e1.

19. Undseth R, Berstad A, Klow NE, et al. Abnormal accumulation of intestinal fluid following ingestion of an unabsorbable carbohydrate in patients with irritable bowel syndrome: an MRI study. Neurogastroenterol Motil 2014;26:1686-93.

20. Pritchard SE, Marciani L, Garsed KC, et al. Fasting and postprandial volumes of the undisturbed colon: normal values and changes in diarrhea-predominant irritable bowel syndrome measured using serial MRI. Neurogastroenterol Motil 2013.

21. Sandberg TH, Nilsson M, Poulsen JL, et al. A novel semi-automatic segmentation method for volumetric assessment of the colon based on magnetic resonance imaging. Abdom Imaging 2015.

22. Savarino E, Savarino V, Fox M, et al. Measurement of oro-caecal transit time by magnetic resonance imaging. Eur Radiol 2015;25:1579-87.

23. Murray K, Wilkinson-Smith V, Hoad C, et al. Differential effects of FODMAPs (fermentable oligo-, di-, mono-saccharides and polyols) on small and large intestinal contents in healthy subjects shown by MRI. Am J Gastroenterol 2014;109:110-9.

24. Spiller RC, Humes DJ, Campbell E, et al. The Patient Health Questionnaire 12 Somatic Symptom scale as a predictor of symptom severity and consulting behaviour in patients with irritable bowel syndrome and symptomatic diverticular disease. Alimentary Pharmacology \& Therapeutics 2010;32:811-820.

25. Suarez FL, Savaiano DA, Levitt MD. A comparison of symptoms after the consumption of milk or lactose-hydrolyzed milk by people with self-reported severe lactose intolerance. New England Journal of Medicine 1995;333:1-4.

26. Nelis GF, Vermeeren MAP, Jansen W. Role of Fructose-Sorbitol Malabsorption in the Irritable Bowel Syndrome. Gastroenterology 1990;99:1016-1020. 
27. Tornblom H, Van Oudenhove L, Sadik R, et al. Colonic transit time and IBS symptoms: what's the link? Am J Gastroenterol 2012;107:754-60.

28. Skoog SM, Bharucha AE, Zinsmeister AR. Comparison of breath testing with fructose and high fructose corn syrups in health and IBS. Neurogastroenterol Motil 2008;20:505-11.

29. Rumessen JJ, Gudmand-Høyer E. Functional Bowel Disease: Malabsorption and Abdominal Distress After Ingestion of Fructose, Sorbitol, and Fructose-Sorbitol Mixtures Gastroenterology 1988;95:694-700.

30. Spiegel BM, Bolus R, Agarwal N, et al. Measuring symptoms in the irritable bowel syndrome: development of a framework for clinical trials. Aliment Pharmacol Ther 2010;32:1275-91.

31. Le Neve B, Posserud I, Bohn L, et al. A combined nutrient and lactulose challenge test allows symptom-based clustering of patients with irritable bowel syndrome. Am J Gastroenterol 2013;108:786-95.

32. Nilsson M, Sandberg TH, Poulsen JL, et al. Quantification and variability in colonic volume with a novel magnetic resonance imaging method. Neurogastroenterology \& Motility 2015;27:1755-1763.

33. Melchior C, Gourcerol G, Dechelotte P, et al. Symptomatic fructose malabsorption in irritable bowel syndrome: A prospective study. United European Gastroenterol J 2014;2:131-7.

34. Berg LK, Fagerli E, Martinussen M, et al. Effect of fructose-reduced diet in patients with irritable bowel syndrome, and its correlation to a standard fructose breath test. Scand J Gastroenterol 2013;48:936-43.

35. Yang J, Deng $\mathrm{Y}$, Chu $\mathrm{H}$, et al. Prevalence and presentation of lactose intolerance and effects on dairy product intake in healthy subjects and patients with irritable bowel syndrome. Clin Gastroenterol Hepatol 2013;11:262-268.e1.

36. Rao SS, Kavelock R, Beaty J, et al. Effects of fat and carbohydrate meals on colonic motor response. Gut 2000;46:205-11.

37. Zhu Y, Zheng X, Cong Y, et al. Bloating and distention in irritable bowel syndrome: the role of gas production and visceral sensation after lactose ingestion in a population with lactase deficiency. Am J Gastroenterol 2013;108:1516-25.

38. Canavan $\mathrm{C}$, Card $\mathrm{T}$, West $\mathrm{J}$. The incidence of other gastroenterological disease following diagnosis of irritable bowel syndrome in the UK: a cohort study. PLoS One 2014;9:e106478. 